\title{
40 ANOS (1978-2018) DA LAGOQUILOSCARÍASE NO PARÁ/BR: CONJECTURAS SOBRE A RELAÇÃO DA DOENÇA COM ASPECTOS SOCIAIS E NATURAIS DA AMAZÔNIA
}

\section{YEARS (1978-2018) OF LAGOCKILLOSARIASIS IN PARÁ / BR: CONJECTURES ON THE RELATION OF DISEASE TO THE SOCIAL AND NATURAL ASPECTS OF AMAZON}

\author{
Darlan Morais Oliveira \\ Universidade Federal do Sul e Sudeste do Pará - PA \\ darlan morais@hotmail.com
}

\begin{abstract}
RESUMO
Objetivo: delinear a distribuição e caracterização da lagoquilascaríase no Pará com base nos casos descritos na literatura. Métodos: Revisão Sistemática da Literatura somada à Revisão Integrativa da Literatura, realizando buscas na base de dados Google Acadêmico, Scielo Brasil, Periódicos Capes, Lilacs, Medline e PubMed de publicações sobre a lagoquilascaríase, entre 1978-2018. Resultados: foram encontradas 17 publicações, as quais constaram 44 casos descritos, oriundos do Pará, sendo que a maioria deles ocorreram em municípios da porção sudeste do estado, principalmente nos de Marabá e Xinguara, e demais municípios entornos. Além disso, ficou nítida uma maior quantidade de casos entre os anos 80 e 90, com queda brusca a partir de 2000, o que possivelmente se justifica pela falta de ocorrência ou de diagnóstico de novos casos. Conclusão: a partir de um melhor delineamento da distribuição de casos em pontos específicos no estado, podem ser elaborados estudos para essas localidades por meio de instituições de ensino e saúde, com o intuito de sanar dúvidas sobre a lagoquilascaríase.
\end{abstract}

Palavras - chave: Doença Negligenciada. Lagochilascaris minor. Sudeste do Pará.

\begin{abstract}
Objective: to outline the distribution and characterization of lagoquilascariasis in Pará based on the cases described in the literature. Methods: Systematic Literature Review plus Integrative Literature Review, performing searches in the Google Scholar, Scielo Brasil, Capes, Lilacs, Medline and PubMed databases of publications on lagoquilascariasis between 1978-2018. Results: 17 publications were found, which described 44 cases from Pará, most of which occurred in municipalities in the southeastern portion of the state, mainly in Marabá and Xinguara, and other surrounding municipalities; also, there was a clear greater number of cases between the 80 s and 90 s, with a sharp drop from 2000 , which possibly is justified by the lack of occurrence or diagnosis of new cases. Conclusion: based on a better delineation of the distribution of cases in specific points in the state, research for these locations can be thought through educational and health institutions, in order to answer questions about lagoquilascariasis
\end{abstract}

Keywords: Neglected Disease. Lagochilascaris minor. Southeast of Pará.

\section{INTRODUÇÃO}

A lagoquilascaríase é uma zoonose causada pelo nematódeo Lagochilascaris minor, sendo uma parasitose rara que não representa risco de grandes epidemias para nenhuma localidade global, no entanto, a maioria de seus casos está restrita às áreas rurais. Além disso, as infecções podem ser reincidentes após aparente cura, ou ainda, ter uma evolução crônica de 5 a 20 anos (REY, 2011; LEÃO, FAIHA NETO, LEÃO, 2017; POÇÔ; CAMPOS, 1998; PALHETA NETO et al, 2002; VASCONCELOS et al, 2017).

Recebido em: 12/04/2020

Aceito para publicação em: 22/12/2020. 
Em termos de distribuição, a lagoquilascaríase está confinada na América Latina, tendo sua primeira ocorrência em Trinidade e Tobago, em 1909. Já no Brasil, registrou-se o primeiro caso em 1968 no estado de São Paulo, porém, a partir de 1978, com o primeiro caso registrado no Pará (PALHETA NETO et al, 2002), seguiram-se diversos relatos da doença chegando a 127 casos mundiais, dentre esses, 98 somente no Brasil e 61 no Pará (LEÃO; FAIHA NETO, 2017).

Há muitas dúvidas que pairam sobre a lagoquilascaríase mesmo já tendo decorrido 100 anos de sua descoberta no mundo, 50 anos no Brasil e 40 anos no Pará. Logo, o estado do Pará, com a maioria dos casos, torna-se um local estratégico para pesquisas que visam sanar dúvidas.

Apesar de tantos anos do descobrimento da doença, ainda persistem algumas incertezas sobre a moléstia, devido à inexistência de um tratamento $100 \%$ efetivo, não sendo definido o seu mecanismo de transmissão e ciclo, sabendo-se apenas que acomete pessoas que, geralmente, moram em áreas remotas, rurais, florestais, predominantemente na região amazônica, sem que haja uma explicação definitiva sobre esse fato (MAGALHÃES, 2019).

Sugere-se que o ciclo da doença envolva roedores, como hospedeiros intermediários, e carnívoros, como hospedeiros definitivos, sendo o homem um hospedeiro acidental. Desse modo, as formas adultas do parasita que deposita ovos no interior do hospedeiro definitivo são eliminadas nas fezes. Uma vez no ambiente, os ovos, ao serem consumidos por roedores como pacas e cutias, desenvolvem-se em formas larvárias, novamente infectando espécies que se alimentam desses roedores, incluindo o homem (REY, 2011; PALHETA NETO, 2002).

No corpo do homem, o agente etiológico em questão migra pelos tecidos, reproduzindo-se e causando autoinfecção, apresentando versatilidade ao albergar cerca de vinte e duas partes do corpo, incluindo estruturas vitais com encéfalo e pulmões, podendo levar o indivíduo a óbito (OLIVEIRA, 2019).

Essas características põem a lagoquilascaríase em destaque no âmbito das helmintoses, pois grande parte delas restringe seu crescimento e reprodução no lúmen do intestino, causando sintomas típicos como anemia, vômitos, desnutrição etc. Desse modo, poucos helmintos têm potencial tão severo quanto o L. minor, a ponto de invadir, desenvolver-se, reproduzir-se e lesionar diversos órgãos humanos.

Por ser uma doença emergente, negligenciada, predominante rural e amazônica, sem notificação compulsória (LEÃO, 2016), não se sabe sua dimensão real no Brasil e no mundo porque, ainda que surjam novos casos, estes não são necessariamente informados aos órgãos de vigilância em saúde, sendo que o principal meio de divulgação e informação dessa enfermidade são as publicações no âmbito médico-acadêmico.

Tais publicações são unânimes em apontar o estado do Pará como o mais prevalente em casos dessa patologia, contribuindo com quase metade da casuística global, consequentemente, é uma localidade de importância crucial para o estudo da doença, e para a análise de fatores que permitam investigar e sanar as grandes lacunas que a englobam. Todavia, aparentemente não existe, nessa Unidade Federativa, algum centro de referência para pesquisas sobre a zoonose em debate.

Nesse contexto, estados com menos ocorrências que o Pará, apresentam maior empenho em pesquisas sobre o parasita, na tentativa de elucidar suas imprecisões, como é o caso do estado de Goiás, que com apenas 1 relato autóctone da doença², possui o Instituto de Patologia Tropical e Saúde Pública - ITPSP como referência mundial em pesquisas e tratamento da lagoquilascaríase (UFG, 2014).

Desse modo, fazem-se necessários estudos específicos sobre essa moléstia no estado do Pará, como meio de investigar e diagnosticar novos casos, bem como divulgar informações de prevenção na sociedade e população vulnerável.

Logo, considerando a importância do estado para essa patologia, questiona-se qual é o perfil da lagoquilascaríase dentro do estado do Pará. Assim, realizou-se a presente investigação com o objetivo de delinear a distribuição e caracterização da lagoquilascaríase no Pará, baseando-se na descrição de casos da doença existentes na literatura.

2 Até 1987 foram apontados cerca de 7 casos de lagoquilascaríase no Goiás, sendo que 6 situavam-se no norte do estado, atual Tocantins, permanecendo apenas 1 caso em território goiano. 


\section{METODOLOGIA}

Utilizou-se a Prática Baseada em Evidências - PBE (ERCOLE; MELO; ALCOFORADO, 2014), adaptando e simplificando etapas da revisão sistemática de literatura somada à revisão integrativa da literatura. Considerando as seis etapas da Revisão Integrativa, mencionadas por Souza, Silva e Carvalho (2010), procedeu-se:

A etapa 1 - de seleção de problemas, que, além do problema principal da pesquisa, criou-se outros tais, como: onde se deram os casos de lagoquilascaríase no Pará?; em quais munícipios houve maior concentração de casos?; quais as contribuições sobre a doença esses estudos trouxeram?; e quais as possíveis relações da doença com os aspectos naturais e sociais do Pará e Amazônia?

A etapa 2 - de estabelecimento de critérios ou busca na literatura. Definiu-se como critério de inclusão: publicações listando um ou mais casos de lagoquilascaríase humana ocorridos no estado do Pará. Quanto aos critérios de exclusão, estabeleceu-se: publicações sobre relatos de casos humanos ocorridos em outros estados ou outros países; pesquisas sobre relatos de lagoquilascaríase animal; revisões que não incluíam detalhamento de casos humanos no corpo do texto.

Logo após as etapas já mencionadas, realizou-se busca na base de dados Google Acadêmico, Scielo Brasil, Periódicos Capes, Lilacs, Medline e PubMed, configurando-se como sites para exibição de trabalhos publicados no período entre 1978 e 2018, associando-se aos seguintes termos indexadores: Lagochilascaris minor AND humana AND relato de caso; lagoquilascaríase AND humana AND relato de caso; lagochilascariase AND humana AND relato de caso; lagochilascariose AND humana AND relato de caso; lagoquilascariase AND estado do Pará.

Nessa etapa, localizaram-se de início 264 trabalhos, sendo: 189 provenientes do Google Acadêmico, 21 dos Periódicos Capes, 15 do Scielo, 18 do Lilacs, 11 do Medline e 10 do PubMed. Nessas pesquisas, aplicou-se uma das fases da Revisão Sistemática da Literatura, consistindo na leitura de títulos e resumos e quando necessário procedeu-se a leitura do corpo do trabalho por inteiro (CASTRO, 2001), selecionando, desse modo, 17 publicações conforme fluxograma a seguir:

Figura 1: Seleção de publicações por revisão sistemática

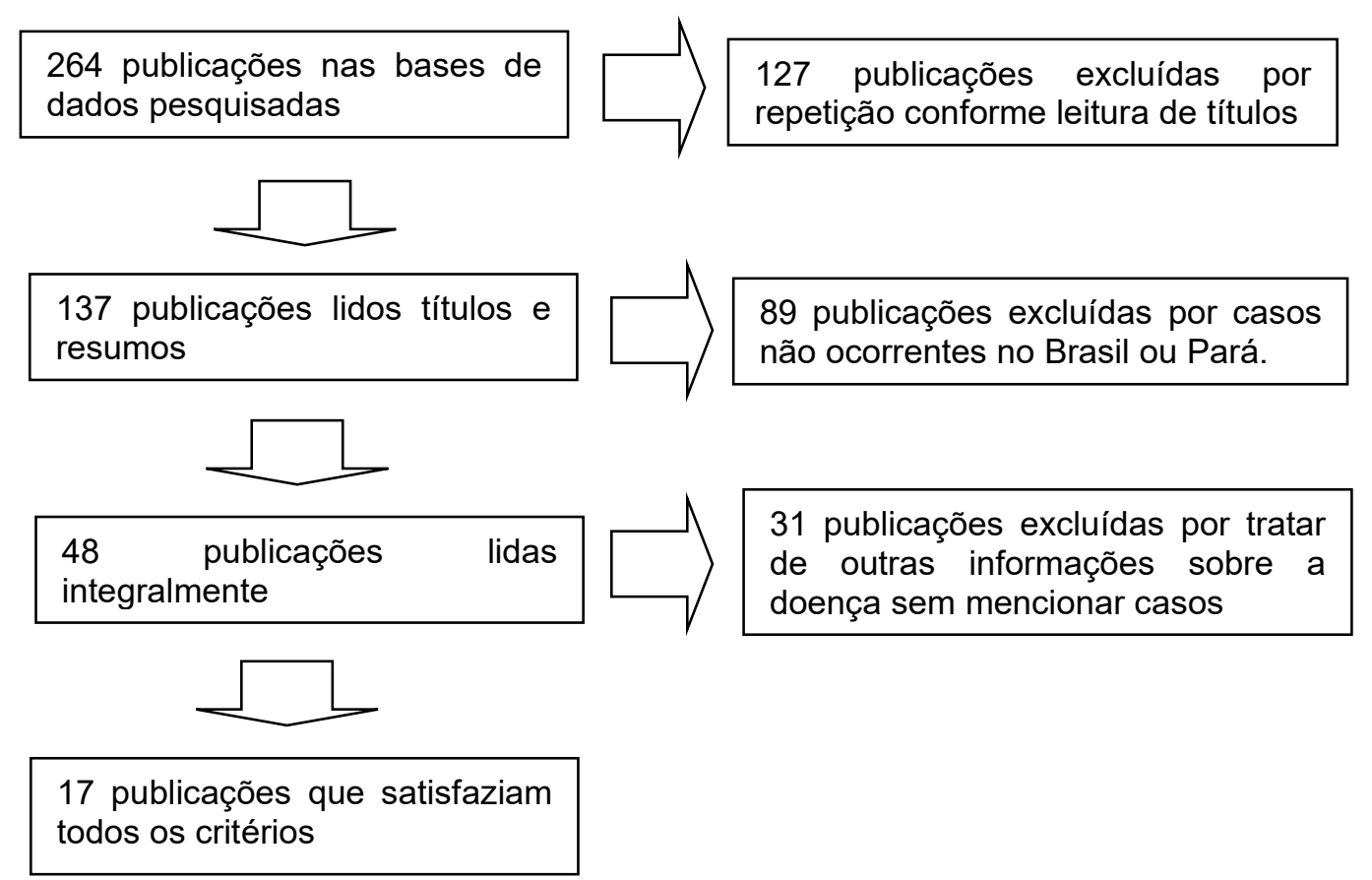

Fonte: A Autoria (2020) 
A etapa 3 - da definição das informações extraídas e categorização dos estudos, optou-se por buscar os municípios onde residiam os pacientes acometidos; quantitativo de casos por município; relações territoriais e naturais entre os municípios; particularidades acerca do paciente, do tratamento e achados que se destacam em relação aos demais casos.

A etapa 4 - referente à avaliação de resultados, procedeu-se com a leitura cuidadosa dos casos notificados nas publicações, averiguando, minuciosamente, situações em que um mesmo caso era descrito em mais de uma publicação, considerando características como idade, sexo e local de origem do paciente, por vezes iniciais do nome e ano de ocorrência do caso.

A etapa 5 - correspondente à interpretação dos resultados, constituem a síntese das informações dispostas na discussão desta pesquisa, conforme a revisão integrativa: "Esta etapa corresponde à fase de discussão dos principais resultados na pesquisa" (MENDES; SILVEIRA; GALVÃO, 2008, p. 762).

O modo como os resultados desses trabalhos foram discutidos, resumidos, estruturados e concluídos, compreende, por fim, a etapa 6 ou etapa final da revisão integrativa, ou etapa de apresentação dos resultados.

\section{RESULTADOS E DISCUSSÃO}

A busca na literatura resultou no encontro de 44 casos de lagoquilascaríase relatados no Pará, o que representa $72 \%$ do total de casos até então descritos no estado, todos relatados em 17 trabalhos científicos publicados entre 1978 e 2018, entre artigos originais, relatos de casos, comunicações em congressos e revisões, os quais foram devidamente numerados por ordem crescente do ano de publicação, conforme a tabela 1 , na sequência: 


\begin{tabular}{|c|c|c|c|c|c|}
\hline $\mathbf{N}^{\circ}$ & Autoria & $\begin{array}{r}\text { Meio de } \\
\text { divulgaçấ }\end{array}$ & & Método & Síntese de resultados \\
\hline 1 & $\begin{array}{l}\text { Leão et al } \\
(1978)\end{array}$ & $\begin{array}{l}\text { Rev. Ins. } \\
\text { Trop. }\end{array}$ & Med. & $\begin{array}{l}\text { Relato de Caso/ } \\
\text { Pesquisa de } \\
\text { Campo/ } \\
\text { Infecções } \\
\text { experimentais }\end{array}$ & $\begin{array}{l}\text { Primeiro registro no estado; ocorrido em Prainha/PA; diagnosticado/tratado em Belém/PA; } \\
\text { Buscas sem sucesso de novos casos no município em questão; Infecção experimental } \\
\text { exitosa em macaco. }\end{array}$ \\
\hline 2 & $\begin{array}{l}\text { Moraes, } \\
\text { Arnaud, Lima } \\
\text { (1983) }\end{array}$ & $\begin{array}{l}\text { Rev. Ins. } \\
\text { Trop. }\end{array}$ & Med. & Relato de Caso & $\begin{array}{l}\text { Descrição de dois casos provenientes de Paragominas/PA e outro município paraense } \\
\text { não identificado situado ao longo da Rodovia Santarem-Cuiabá; diagnosticados/tratados } \\
\text { em Belém/PA. }\end{array}$ \\
\hline 3 & $\begin{array}{l}\text { Rocha, Fraiha } \\
\text { Neto e Barreto } \\
\text { Neto (1984) }\end{array}$ & Hiléia Med. & & $\begin{array}{l}\text { Relato de Caso/ } \\
\text { Revisão } \\
\text { Narrativa }\end{array}$ & $\begin{array}{l}\text { Detalhamento de caso ocorrido em Marabá/PA, e diagnosticado/tratado em Belém/PA, } \\
\text { onde a paciente e o gato de sua residência estavam infectados; Menção de um caso } \\
\text { oriundo de Jacundá/PA; Relato ainda dos três casos descritos nos estudos } 1 \text { e } 2 \text {. }\end{array}$ \\
\hline 4 & $\begin{array}{l}\text { Moraes et al, } \\
\text { (1985) }\end{array}$ & $\begin{array}{l}\text { Rev. Ins. } \\
\text { Trop. }\end{array}$ & & Relato de Caso & $\begin{array}{l}\text { Óbito devido à lagochilascariase pulmonar; paciente oriunda de Curralinho/PA, } \\
\text { diagnosticada/atendida em Belém/PA }\end{array}$ \\
\hline 5 & $\begin{array}{l}\text { Frainha et al } \\
\text { (1986) }\end{array}$ & $\begin{array}{l}\text { Mem Inst } \\
\text { Cruz }\end{array}$ & Osw. & $\begin{array}{l}\text { Revisão } \\
\text { Narrativa }\end{array}$ & $\begin{array}{l}\text { Listagem de catorze casos oriundos do Pará, sendo } 6 \text { casos já relatados nos estudos } \\
1,2,3 \text { e } 4 \text {, os demais casos provinham de: Cametá, São João do Araguaia, Portel, São } \\
\text { Geraldo do Araguaia, Altamira, Conceição do Araguaia e Redenção }\end{array}$ \\
\hline 6 & $\begin{array}{l}\text { Campos et al, } \\
\text { (1987) }\end{array}$ & Rev. Pat. Tro & & $\begin{array}{l}\text { Revisão } \\
\text { Narrativa/ } \\
\text { Infecções } \\
\text { Experimentais }\end{array}$ & $\begin{array}{l}\text { Descrição de três casos oriundos de Conceição do Araguaia/PA, Redenção/PA e } \\
\text { Xinguara/PA, todos diagnosticados no Goiás. Infecções experimentais inexitosas em } \\
\text { camundongos e macacos. }\end{array}$ \\
\hline 7 & $\begin{array}{l}\text { Habid, Leão e } \\
\text { Costa (1989) }\end{array}$ & Zoon. Rev. In & & $\begin{array}{l}\text { Revisão } \\
\text { Narrativa }\end{array}$ & $\begin{array}{l}\text { Descrição de vinte e seis casos ocorridos no Pará, sendo os seis casos anteriores } \\
\text { mencionados no estudos n.os } 1,2,3 \text { e } 4 \text {; e outras vinte novas descrições oriundas de: } \\
\text { Jacundá, Cametá; São João do Araguaia; Marabá; Pacajá; São Geraldo do Araguaia; } \\
\text { Altamira; Tucumã; Rondon; Breves; Portel; Xinguara; Tucuruí; Conceição do Araguaia. }\end{array}$ \\
\hline 8 & $\begin{array}{l}\text { Santos et al, } \\
(1990)\end{array}$ & $\begin{array}{l}\text { An. } \\
\text { Dermato. }\end{array}$ & Bras. & Relato de Caso & $\begin{array}{l}\text { Descrição de paciente oriundo de Marabá/PA tendo transitado por cerca de dois anos por } \\
\text { centros médicos de Marabá/PA e Teresina/PI, passando por diversos exames e } \\
\text { tratamentos até finalmente ser diagnosticado com lagochilascariase no DF onde fora } \\
\text { tratado. }\end{array}$ \\
\hline
\end{tabular}




\begin{tabular}{|c|c|c|c|c|}
\hline 9 & $\begin{array}{l}\text { Veloso et al } \\
(1992)\end{array}$ & $\begin{array}{l}\text { Rev. Ins. Med. } \\
\text { Trop. }\end{array}$ & Relato de Caso & $\begin{array}{l}\text { Três casos, sendo um já mencionado no estudo } 5 \text {, e dois novas descrições: Curionópolis } \\
\text { e Marabá, sendo a última resultante em óbito por infecção no encefalo }{ }^{3} \text {; ambos } \\
\text { diagnosticados no DF. }\end{array}$ \\
\hline 10 & $\begin{array}{l}\text { Campos et al } \\
(1995)\end{array}$ & Rev. Pat. Trop. & Relato de Caso & Caso proveniente de Xinguara/PA, diagnosticado/tratado em Araguaína/TO \\
\hline 11 & $\begin{array}{l}\text { Poçô } \\
\text { Campos } \\
(1998)\end{array}$ & Rev. Pat. Trop. & $\begin{array}{l}\text { Revisão } \\
\text { Narrativa }\end{array}$ & $\begin{array}{l}\text { Vinte e um casos provenientes do Pará, dos quais doze já foram citados nos trabalhos nº } \\
1,2,3,4,5 \text { e } 7 \text {; novas descrições provenientes de: Xinguara, Prainha, Marabá, Rio Maria, } \\
\text { Tucumã, Tailandia e um município indefinido; }\end{array}$ \\
\hline 12 & $\begin{array}{l}\text { Paula et al } \\
(1998)\end{array}$ & Rev. Pat. Trop & Relato de Caso & Caso proveniente de São Domingos/PA, diagnosticado/tratado em Araguaína/TO \\
\hline 13 & $\begin{array}{l}\text { Vieira et al } \\
(2000)\end{array}$ & $\begin{array}{l}\text { Rev. Soc. Bras. } \\
\text { Med. Trop. }\end{array}$ & Relato & $\begin{array}{l}\text { Detalhamento de um dos casos já descrito no estudo n } 9 \text {; originário de Xinguara/PA, } \\
\text { diagnosticado/tratado em Goiânia/GO }\end{array}$ \\
\hline 14 & Leão (2016) & $\begin{array}{l}6^{\circ} \quad \text { INFECTO } \\
\text { Norte-Nordeste }\end{array}$ & $\begin{array}{l}\text { Revisão } \\
\text { Narrativa }\end{array}$ & $\begin{array}{l}\text { Menções aos casos descritos nos estudos } 1,5 \text { e } 7 \text { e menção a um caso proveniente de } \\
\text { Pacajá/PA não descrito nos estudos anteriores. Alegação da eficiência de Levamisol e } \\
\text { Cambendazol no tratamento. }\end{array}$ \\
\hline 15 & $\begin{array}{l}\text { Esper et al, } \\
2017\end{array}$ & $52^{\circ}$ MEDTROP & Relato de Caso & $\begin{array}{l}\text { Caso proveniente de Aveiro/PA, diagnosticado/tratado em Santarém/PA; paciente gestante } \\
\text { com infecção reincidente }\end{array}$ \\
\hline 16 & $\begin{array}{l}\text { Maquiné et al } \\
(2018)\end{array}$ & $\begin{array}{l}\text { Rev Soc Bras } \\
\text { Clin Med. }\end{array}$ & Relato de Caso & $\begin{array}{l}\text { Caso proveniente de Itaituba/PA, diagnosticado/tratado em Manaus/AM; paciente } \\
\text { gestante }{ }^{4} \text {. }\end{array}$ \\
\hline 17 & $\begin{array}{l}\text { Barreto et al } \\
(2018)\end{array}$ & $\begin{array}{l}\text { J Bras Patol Med } \\
\text { Lab }\end{array}$ & Relato de Caso & Caso proveniente de Parauapebas/PA, diagnosticado/tratado em Belo Horizonte/MG. \\
\hline
\end{tabular}

Fonte: A Autoria (2020)

3 Primeiro caso da enfermidade e o primeiro óbito no estado, ocorrido em 1974, embora tenha sido publicado 18 anos após sua ocorrência, quando já haviam sido relatadoss outros casos e um óbito.

$4 \quad$ Primeiro caso em gestante, tendo se dado em 1987, porém somente fora divulgado 30 anos depois, quando já houvera publicação do $2^{\circ}$ ocorrido em gestante.
DOI: http://dx.doi.org/10.14393/Hygeia17053741
Hygeia
v.17
p. 1 - 13,
2021
página 6 
Da leitura atenciosa das publicações, analisou-se as relações de tempo (anos de ocorrência) e de espaço geográfico, ou seja, os municípios e regiões geográficas oficiais de onde partiam os casos, entretanto, em muitas situações, o paciente residiu em mais de um município paraense, nesse caso, foi considerado o último município de residência do indivíduo, resultando no disposto da tabela 2 a seguir:

Tabela 2: Distribuição de casos de Lagoquilascaríase no Pará por região geográfica e municípios

\begin{tabular}{|c|c|c|c|}
\hline UF & $\begin{array}{c}\text { Região } \\
\text { Intermediária } \\
\text { (IBGE) }\end{array}$ & Município & $\begin{array}{l}N^{\circ} \text { de } \\
\text { Casos }\end{array}$ \\
\hline \multirow{33}{*}{$\frac{\mathbb{x}}{\frac{1}{\alpha}}$} & \multirow{11}{*}{ MARABÁ } & Marabá & 7 \\
\hline & & São João do.Araguaia & 2 \\
\hline & & Tucuruí & 2 \\
\hline & & Parauapebas & 1 \\
\hline & & Curionópolis & 1 \\
\hline & & São Gerạidọ & 1 \\
\hline & & São Domingos & 1 \\
\hline & & Jacundá : & 1 \\
\hline & & Rondon & 1 \\
\hline & & Pacajá & 1 \\
\hline & & SUBTOTAL & 18 \\
\hline & \multirow{6}{*}{ REDENÇÃO } & Xinguara & 6 \\
\hline & & Tucumã & 2 \\
\hline & & Conceição do Araguaia & 2 \\
\hline & & Redenção & 1 \\
\hline & & Rio Maria & 1 \\
\hline & & SUBTOTAL & 12 \\
\hline & \multirow{4}{*}{ BREVES } & Portel & 2 \\
\hline & & Curralinho & 1 \\
\hline & & Breves & 1 \\
\hline & & SUTOTAL & 4 \\
\hline & \multirow{4}{*}{ SANTATÉM } & Prainha & 2 \\
\hline & & Aveiro & 1 \\
\hline & & Itaituba & 1 \\
\hline & & SUBTOTAL & 4 \\
\hline & \multirow{3}{*}{ BELÉM } & Tailândia & 1 \\
\hline & & Cametá & 1 \\
\hline & & SUBTOTAL & 2 \\
\hline & CASTANHAL & Paragominas & 1 \\
\hline & ALTAMIRA & Altamira & 1 \\
\hline & \multirow{2}{*}{\multicolumn{2}{|c|}{$\begin{array}{l}\text { Município não especificado (Rod. Santarém/Cuiabá) } \\
\text { Município não especificado }\end{array}$}} & 1 \\
\hline & & & 1 \\
\hline & \multicolumn{2}{|c|}{ TOTAL } & 44 \\
\hline
\end{tabular}

Fonte: A Autoria (2020)

Grande parte das publicações consistiam em curtos relatos descrevendo o tratamento, perfis dos pacientes e quadros clínicos. Estes eram caracterizados principalmente por lesões (exsudados, purulência, fístulas, tumoração, inchaço) no pescoço, ouvido e mastoide, por vezes também nos pulmões e sistema nervoso central. Os pacientes apresentavam duas características em comum, detectadas na anamnese: habitavam na zona rural e alimentavam-se de carne de caça. Quanto ao tratamento, eram usados anti-helminticos (cambendazol, levamisol, dealbendazol, ivermectina) geralmente associados. Todas essas confluências estão de acordo com os argumentos de Palheta 
Neto et al (2002) e Leão, Faiha Neto e Leão (2017) que descrevem os quadros clínicos, epidemiologia e tratamento dessa doença.

A maioria dos estudos encontrados não traz divergências ou grandes destaques no perfil da doença, os casos ocorridos no Pará coincidem com as descrições de outros ocorridos em diferentes partes do país e do mundo.

Contudo, deram importantes contribuições acerca do ciclo da doença descritos nos estudos de Leão et al al (1978) e Rocha, Fraiha e Barreto (1984) que relatam infecções experimentais e naturais, indicando o ciclo digenético do parasita que tem o homem e os felinos como hospedeiros definitivos, algo que fora experimentalmente sugerido por Barbosa, Barbosa e Campos (2005) e naturalmente encontrado por Reis et al (2011).

Também, destacou-se o uso de cambendazol e levamisol no tratamento da doença, como relatado no estudo $n^{\circ}$ Leão (2014). Além da descrição de dois casos de infecção em pacientes grávidas (ESPER et al, 2017; MAQUINÉ et al, 2018), talvez os únicos casos na epidemiologia da doença, o que chama a atenção pelo possível risco existente nessa condição, haja vista que o $L$. minor pode albergar mamas e órgãos internos próximos ao útero, como ovários (PALHETA NETO et al 2002; ESPER et al, 2017).

Da análise geográfica, as áreas com mais incidência estão nas regiões imediatas ${ }^{5}$ de Marabá e Redenção (áreas próximas que fazem divisa uma com a outra, juntas, anteriormente, formavam a Mesorregião do Sudeste do Pará). Trata-se de locais em que a literatura aponta como sendo o ponto de maior concentração de casos (LEÃO, FAIHA NETO, LEÃO, 2017), e também onde ocorre a confluência dos rios Araguaia e Tocantins, concordando assim com o argumento de Poçô e Campos (1998) que sugere que nessa localidade o L. minor teria sua ecologia ideal.

Dentre essas duas regiões, Marabá e Xinguara são os dois municípios que concentram mais ocorrências que os demais, juntos somam 13 ocorrências, com aproximadamente $10 \%$ dos casos mundiais, $13 \%$ dos nacionais e $21 \%$ no estado. Logo, são valores expressivos pois a patologia está distribuída em toda a América Latina (LEÃO, FAIHA NETO, LEÃO, 2017), valores que podem ser ainda maiores, considerando que não foram encontrados, nesta investigação, todos os 61 casos ocorridos no estado.

Os referidos municípios estão próximos um do outro e estão situados na porção Sudeste Paraense, juntamente com os demais municípios que compõem as regiões intermediárias de Marabá e Redenção, totalizando 30 ocorrências encontradas nesta pesquisa para essa área territorial, ficando nítida a concentração de casos e corroborando com a literatura, conforme se observa na figura 2 adiante, onde cada estrela indica a localização de cada caso encontrado neste estudo:

Da análise temporal, verificou-se o primeiro caso em 1974 (VELOSO et al, 1992) publicado dezoito anos após, e o último ocorrera 42 anos depois em 2016 em Aveiro/PA (ESPER et al, 2017). No entanto, as últimas publicações da doença no estado foram em 2018 narrando um caso ocorrido 30 anos antes, 1987 (MAQUINÉ et al, 2018) e outro caso ocorrido em 2015 (BARRETO et al, 2018).

Ainda sobre a análise temporal dos casos, nota-se pela tabela 1 que a maior parte deles ocorreram entre as décadas de 80/90, com significativa redução durante a década de 2000/10, nas quais apenas os casos descritos por Esper et al (2017) e Barreto et al (2018) ocorreram na atualidade, as demais notificações publicadas nesse período tratavam-se de revisões narrativas de ocorrências anteriores ou relatos de casos decorridos há muitos anos antes, havendo, portanto, estagnação de novos casos nos últimos tempos.

Essa constatação vai ao encontro das alegações de Leão (2016), demonstrando que $72,6 \%$ dos casos da doença no mundo se deram entre 1980-1999, tendo queda vertiginosa a partir de $2000 \mathrm{com}$ menos de $10 \%$ das ocorrências, apontando as possíveis causas: ausência de casos novos (a doença deixou de ocorrer); falta de notificação e de interesse em divulgar novos casos (eles são diagnosticados e tratados, mas não são comunicados); e falta de diagnóstico por desconhecimento da doença.

\footnotetext{
${ }^{5}$ Atual subdivisão dos estados proposta pelo IBGE em 2017 em substituição às mesorregiões.
} 
Figura 2: Localização de casos de lagoquilascaríase no estado do Pará encontrados neste estudo

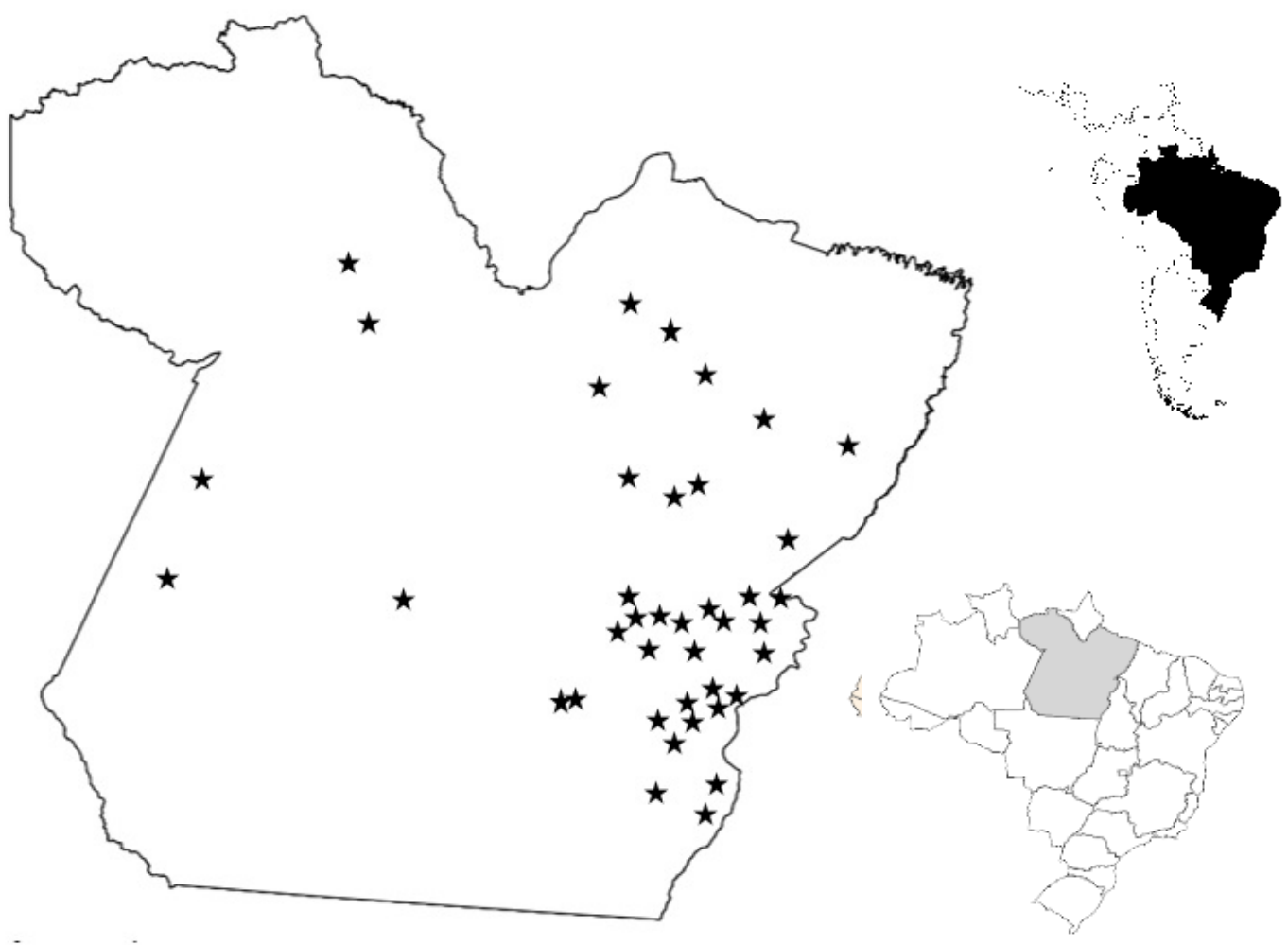

Fonte: A Autoria (2020)

É possível conjecturar cada uma dessas possibilidades, levando-se em conta os aspectos sociais e naturais no estado do Pará e Região Amazônica que podem fornecer perspectivas plausíveis para a dinâmica epidemiológica dessa rara enfermidade.

Cita-se como exemplo o êxodo rural ocorrido nas últimas décadas onde, entre 1991 e 2000, na região Norte $23,7 \%$ da população rural ao migrou, abandonando o campo, sendo o maior índice em seis períodos (ALVES et al, 2011). Nesse contexto, o estado do Pará possuía em 1991 uma equiparação entre a população urbana e rural com aproximados 2,5 e 2,3 milhões de habitantes respectivamente, enquanto que em 2010 a diferença era de 5,1 milhões para a área urbana e 2,3 milhões para a rural (IBGE, 1991; IBGE, 2010), logo a população rural em números se estagnou mas em proporção caiu, uma consequência disso é que com menos pessoas no campo, menor a possibilidade de haver infecções atreladas a ambientes rurais como é o caso da lagoquiláscariase.

Outro possível fato para a ausência de novos casos é a melhora do saneamento básico, ocorrida também nos últimos anos. Se comparado às décadas anteriores, no Pará em específico, ente 19802000 , a taxa de domicílios com água tratada subiu de $40 \%$ para quase $50 \%$, enquanto que o acesso à rede de esgoto subiu de $0 \%$ a $10 \%$ (SAIANI; TONETO JUNIOR, 2010). A helmintose em questão é comum em pessoas vivendo à margem da pobreza e em péssimas condições de saneamento, sem acesso à água tratada, como foram descritos nos por Rocha, Fraiha Neto e Barreto Neto (1984), Santos et al (1990), Campos et al (1995) e Vieira et al (2000) e portanto, presume-se que melhorando o saneamento reduzem-se as chances da infecção.

Também é pertinente enfatizar mais uma vez que, o hipotético ciclo da doença envolve o consumo de carne de roedores silvestres, diante disso, caso esse consumo seja reduzido, possivelmente diminuiria os números de infecções, o que também é passível de estar ocorrendo, tendo em vista alguns estudos apontarem que populações rurais amazônicas tiveram redução na disponibilidade de carne de caça na atualidade, em decorrência de atividades predatórias antrópicas no ambiente natural, alterando os hábitos alimentares da população (NASCIMENTO; GUERRA, 2016; RAMOS et 
al, 2019), alterações essas que também se justificam pelo acesso às cidades e ao assistencialismo de políticas públicas que facilitam a introdução de novas fontes proteicas, como alimentos industrializados às áreas remotas de comunidades caboclas amazônicas (SILVA; GARAVELLO, 2012) reduzindo assim a caça.

Todavia, fatos como melhoras nas condições de saneamento da polução rural, o êxodo rural, e a mudança de hábitos alimentares ainda são sutis no Estado Pará, logo, ainda existem grandes áreas no estado sem saneamento, muitos habitantes na zona rural e o tradicional costume de comer carne de caça. Diante disso, a possibilidade da redução de casos de lagoquilascaríase no estado, no país e no mundo é mais provável pela falta de diagnóstico da doença e consequente falta de notificações/divulgações da mesma do que pelo fato de não mais haver infecções.

Várias situações corroboram para que as infecções ainda estejam acontecendo sem o devido diagnóstico. Inicialmente, frisa-se que as condições do Pará e Amazônia propiciam para a manutenção da doença, pois as características ambientais e sociais da Amazônia brasileira em si são determinantes para os quadros epidemiológicos e assistência à saúde local, características tais como: extensa área territorial e difíceis vias de acesso, além da grande diversidade biológica e social (CONFALONIERI, 2005).

$\mathrm{O}$ aspecto natural implica bastante na ocorrência de doenças infecciosas e parasitárias, bem como acidentes por animais peçonhentos decorrentes do contato humano com o meio natural (vegetação; solo; animais; água etc.), especialmente nas comunidades tradicionais, por sua exposição constante às espécies peçonhentas, pela ingestão de carne de espécies silvestres e pelo contato com animais domésticos que aumentam o risco de infecções, na qual se inclui a lagoquilascaríase (CONFALONIERI, 2005).

Esses argumentos reforçam a hipótese de que ainda existem casos dessa moléstia no Pará e Amazônia Legal, entretanto, a vasta territoriedade e pouca densidade demográfica gerando relativo isolamento de comunidades rurais imersas aos perigos naturais do ecossistema local, fazem com o acesso à assistência, saúde e o diagnóstico se torne dificultosos.

A questão do correto diagnóstico é algo discutível no contexto da lagoquilascaríase, uma vez que a mesma é confundida com paracoccidioidomicose, actinomicose, leishmaniose, tuberculose ganglionar, etc, (REY, 2011), doenças também comuns na área endêmica em questão, gerando confusão no diagnóstico o que dificulta a notificação de novos casos confirmados.

As pesquisas de Moraes et al (1985), Santos et al (1990) e Paula et al (1998) mostraram que foram realizados nos pacientes exames para diagnóstico de tuberculose, infecções micóticas, infecções bacterianas, doenças de Chagas e toxoplasmose, sugerindo assim confusão de sinais e sintomas da lagoquilascaríase com essas doenças.

Nesse sentido, não há como descartar a possibilidade de diagnóstico equivocado da enfermidade em discussão, tanto que Mendes (2018) teoriza que há negligência dos profissionais diante um caso de lagoquilascaríase, e que mortes humanas nas áreas endêmicas, podem ter decorrido de um diagnóstico falso-negativo desta zoonose.

De fato, há indícios da falta de familiaridade dos profissionais de saúde com essa patologia no estado do Pará, pois algumas das publicações elencadas neste estudo relatam que o diagnóstico correto ocorreu em outros estados, mesmo que em alguns casos o paciente tenha procurado antes assistência médica no Pará, isso foi percebido nos trabalhos de Santos et al (1990), Campos et al (1995), Paula et al (1998), Vieira et al (2000), Maquiné et al (2018) e Barreto et al (2018).

Ante esses argumentos, não se descarta a hipótese de que haja mais casos, tanto veterinários quanto humanos, mas eles não estão sendo devidamente diagnosticados e publicados. Nesse sentido, considerando que não é uma doença de notificação compulsória, os meios oficiais de divulgação de casos são as publicações acadêmico-científicas ocorrentes em eventos, periódicos e livros. Sendo assim, compete às instituições de saúde e ensino se dedicarem às pesquisas voltadas para lagoquilascaríase.

Ressalta-se que, instituições de ensino devem realizar pesquisas sobre doenças negligenciadas, tanto que documentos de órgãos internacionais como a Organização Mundial de Saúde (OMS) e Organização Pan-Americana da Saúde (OPAS) têm recomendado a importância da temática dessas 
doenças no espaço escolar (ASSIS; JORGE, 2018), portanto, por equivalência, isso também se aplica a qualquer nível de ensino, seja ele formal ou informal, visando manter a sociedade informada e prevenida, especialmente a população mais vulnerável a essas doenças.

Nesse contexto, atualmente a porção sudeste do Pará mais afetada pela enfermidade, conta com grandes centros urbanos, onde existem instituições acadêmicas próprias da região, públicas e privadas, com cursos na área da saúde, montando assim um cenário propício para o desenvolvimento de pesquisas locais que podem clarificar as questões que envolvem essa doença.

\section{CONCLUSÃO}

Este estudo permitiu delinear cerca de $70 \%$ da distribuição da lagoquilascaríase no Pará, além de relacionar e analisar perfis, distribuição temporal e espacial das ocorrências, permitindo fazer inferências sobre a epidemiologia da doença no estado e conjecturas sobre sua situação atual.

Esta investigação ratifica o que já consta na literatura, descrevendo seu suposto ciclo, sinais e sintomas, e seu perfil epidemiológico, confirmando sua prevalência no sudeste do Pará, configurando então essa parte do estado como alvo promissor para estudos sobre a doença.

Além disso, essa pesquisa traz como novidade o melhor delineamento e detalhamento da distribuição geográfica da doença no Pará, apontando pontos mais específicos de ocorrência, onde poderão ser pensadas futuras estratégias para o desenvolvimento de novas pesquisas.

Para que ocorram novas pesquisas, recomenda-se que por meio das instituições de ensino e pesquisa (UNIFESSPA, UEPA, IFPA e outras) e de saúde, situadas nas áreas de maior incidência, no caso o Sudeste do Estado, nos munícipios de Marabá e Xinguara por exemplo, desenvolvam-se grupos de estudo, investigações de campo, programas de prevenção e notificação locais, dentre outras medidas pertinentes. Tudo isso no intuito de descobrir novos possíveis casos e preencher lacunas como o ciclo de vida exato do $L$. minor, o diagnóstico oportuno e tratamento mais eficiente.

\section{REFERÊNCIAS}

ALVES, E. et al. Êxodo e sua contribuição à urbanização de 1950 a 2010. Revista da Política Agrícola, v. 20, n. 2, p. 80-88, 2011

ASSIS, S. S.; JORGE, T. C. O que dizem as propostas curriculares do Brasil sobre o tema saúde e as doenças negligenciadas?: aportes para a educação em saúde no ensino de ciências Ciênc. Educ., v. 24, n. 1, p. 125-140, 2018. https://doi.org/10.1590/1516-731320180010009

BARBOSA, C.A.L; BARBOSA, A.P; CAMPOS, D.M.B. Gato doméstico (Felis catus domesticus) como possível reservatório de Lagochilascaris minor LEIPER (1909). Revista De Patologia Tropical, v. 34, n. 3, p. 205-211, 2005

BARRETO, L.F. Lagochilascariasis: case report. J Bras Patol Med Lab v. 54, n. 4, p. 245-248, 2018

CAMPOS, D.M.B et al. Lagochilascaríase humana. Registro de um novo caso procedente do sul do Pará. Revista Patologia Tropical. v.24, n.2, p. 313 322, 1995. https://doi.org/10.5935/16762444.20180042

CASTRO, A.A. Revisão sistemática: identificação e seleção dos estudos. In: CASTRO, A.A. Revisão sistemática com e sem metanálise. São Paulo: AAC; 2001. Disponível em: http://www.metodologia.org. Acesso em 12 abr. 2019.

CONFALONIERI, U.E.C. Saúde na Amazônia: um modelo conceitual para a análise de paisagens e doenças. Estudos Avançados, v. 19, n. 53, p. 221-236, 2005. https://doi.org/10.1590/S0103$\underline{40142005000100014}$

ERCOLE, F.F; MELO, L.S; ALCOFORADO, C.L.G.C. Revisão Integrativa versus Revisão Sistemática. Rev Min Enferm. v.18, n.1, p 9-11, 2004

ESPER, H.R.; PINHEIRO, O.C.; QUIROGA, M.; ASSY, J.G.P.L. Lagoquilascaríase na gravidez: relato de caso de helmintíase emergente na Região Amazônica. In: Congresso Brasileiro de Medicina Tropical, 52, 2016, Maceió. Resumos do congresso ..., Maceió, Sociedade Brasileira de Medicina Tropical, 2016.

DOI: http://dx.doi.org/10.14393/Hygeia17053741 $\quad$ Hygeia $\quad$ v.17 $\quad$ p. 1-13, $2021 \quad$ página 11


FRAIHA NETO, H.; LEÃO R.N.Q; COSTA, F.S.A. Lagoquilascaríase humana e dos animais domésticos. Zoon. Revista Internacional, v.1, n.1, p. 25-33, 1989.

FRAIHA, $\mathrm{H}$ et al. Lagoquilascaríase. In: Instituto Evandro Chagas: 50 anos de contribuição às ciências biológicas e a medicina tropical. Belém: Fundação Serviços de Saúde Pública, 1986.

IBGE - Instituto Brasileiro de Geografia e Estatística. Brasil/Pará. Disponível em: https://cidades.ibge.gov.br/brasil/pa/panorama. Acesso em: 18 ago 2019.

IBGE - Instituto Brasileiro de Geografia e Estatística. CENSO DEMOGRÁFICO 1991: Resultados do universo relativos às características da população e dos domicílios. n. 7. SEPLAN/IBGE: Rio de Janeiro, 1991.

LEÃO, R.N.Q et al. Infecção humana pelo Lagochilascaris minor Leiper, 1909. Registro de um caso observado no Estado do Pará (Brasil). Revista do Instituto de Medicina Tropical. São Paulo, v.20, n.5, p. 300-306, 1978

LEÃO, R.N.Q. Lagochilascaríase. In: CONGRESSO NORTE-NORDESTE DE INFECTOLOGIA, 6., 2016, São Luis. Comunicação Oral do Congresso.... São Luis: Sociedade Brasileira de Infectologia, 2016. Disponível em: https://www.youtube.com/watch?v=FwzaNwg8jxw\&t=1168s. Acesso em: 25 jun 2019

LEÃO, R.N.Q; FRAINHA NETO, H; LEÃO, A. Dez perguntas sobre lagoquilascaríase. Boletim da Sociedade Brasileira de Infectologista, s.v, s.n, p. 18-19, jun, 2017.

MAGALHÃES, C. Doenças que ocorrem no mundo se comportam diferente na Amazônia. Disponível em: https://www.oliberal.com/para/doencas-que-ocorrem-no-mundo-se-comportamdiferente-na-amazonia-1.192005. Publicado em: 11 set 2019. Acesso em: 4 out 2019.

MAQUINÉ, G.A et al. Lagoquilascaríase humana: um diagnóstico a ser lembrado diante de tumoração de cabeça e pescoço: relato de caso. Revista da Sociedade Brasileira Clinica Medica. v.16, n.1, p. 45-47, 2018.

MENDES, K.D.S; SILVEIRA, R.C.C.P; GALVÃO, C.M. Revisão integrativa: método de pesquisa para a incorporação de evidências na saúde e na enfermagem. Texto Contexto Enferm, v 17, n. 4, p. 758-764, 2008. https://doi.org/10.1590/S0104-07072008000400018

MENDES, T.C et al. Lagoquilascaríase humana - enfoque no diagnóstico clínico-laboratorial. In: Congresso Brasileiro de Ciências Da Saúde, 3, 2018, Campina Grande. Anais Do Evento... Campina Grande, UEPB, 2018.

MORAES, M.A.P et al. Infecção pulmonar fatal por Lagochilascaris sp, provavelmente Lagochilascaris minor Leiper, 1909. Revista do Instituto de Medicina Tropical. v.27, [s. n.], p. 46-52, 1985. https://doi.org/10.1590/S0036-46651985000100009

MORAES, M.A.P et al. Novos casos de infecção humana por Lagochilascaris leiper, minor 1909, encontrados no estado do Pará, Brasil. Revista Instituto de Medicina Tropical. v. 25, n.3, p.139146, 1983.

NASCIMENTO, E.C; GUERRA.; G.A.D.Do avortado ao comprado: práticas alimentares e a segurança alimentar da comunidade quilombola do baixo Acaraqui, Abaetetuba, Pará. Bol. Mus. Para. Emílio

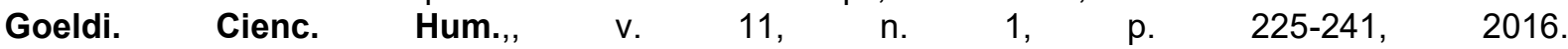
https://doi.org/10.1590/1981.81222016000100012

PALHETA NETO, F. et al. Contribuição ao estudo da lagoquilascaríase humana. Revista Brasileira de Otorrinolaringologia, v. $68, \quad$ n.1, p. 101-106, 2002 . https://doi.org/10.1590/S0034$\underline{72992002000100018}$

PAULA, J.B et al. Relato de um novo caso de lagochilascaríase humana procedente do sul do Pará. Revista de Patologia Tropical, v.27, n.l, p. 71-76, 1998.

POÇÔ, J.M; CAMPOS, D.M.B. Lagochilascaris Núnor Leiper, 1909: Nove Décadas de Revisão Bibliográfica. Revista de Patologia Tropical. v.27, n.1, p. 11-34. jan-jun. 1998. https://doi.org/10.5216/rpt.v27i1.17193 
RAMOS, R.M et al. Caça e uso da fauna. Disponível em: https://www.researchgate.net/publication/257328583 Caca e uso da fauna. Acesso em: 02 set 2019.

REIS, R. et al. Lagochilascaris minor (Nematoda, Ascarididae) em gato doméstico: relato de caso. Veterinária em Foco, Canoas, v.9, n. 1, p. 43-48, jul./dez. 2011.

REY, L. Parasitologia. 4ª ed. Rio de Janeiro: Guanabara Koogan, 2011.

ROCHA, M.P.C.; FRAIHA NETO, H. E BARRETO NETO, A.C. Infecção de ouvido médio e mastoide por Lagochilascaris minor Leiper, 1909 (Nematoda, Ascarididae). Relato de uma caso do Sul do Estad do Pará, Amazonia, Brasil. Hiléia Médica, v. 6, n 1/2, p. 3-14, 1984

SAIANI, C.C.S; TONETO JÚNIOR, R.. Evolução do acesso a serviços de saneamento básico no Brasil (1970 a 2004). Economia e Sociedade, v. 19, n. 1, p. 79-106, 2010. https://doi.org/10.1590/S0104-06182010000100004

SANTOS, V.M et al. Relato de caso de infecção humana por Lagochilascaris minor. Anais Brasileiros de Dermatologia, v.65, n.4, p. 4p., 1990. Disponível em: http://www.anaisdedermatologia.org.br/detalhe-artigo/788/Relato-de-caso-de-infeccao-humana-porLagochilascaris-minor. Acesso em: 12 jul 2019.

SILVA; R.J; GARAVELLO, M.E.P.E. Ensaio sobre transição alimentar e desenvolvimento. Segurança Alimentar e Nutricional, v. 19, n. 1, p.1-7, 2012. https://doi.org/10.20396/san.v19i1.8634664

SOUZA, M.T; SILVA, M.D; CARVALHO, R. Revisão integrativa: o que é e como fazer. Einstein. v $8, n$. 1, p102-106, 2010.

UFG - Universidade Federal de Goiás. Médico do IPTSP (UFG) apresenta casos clínicos de Lagochilascaríase. Disponível em <https://www.ufg.br/n/58759-medico-do-iptsp-ufg-apresentacasos-clinicos-de-lagochilascariase> . Publicada em: 14 jul 2011. Atualizada em: 21 ago 2014. Acesso em: 24 jul 2019.

VELOSO, M.G.P. et al. Lagoquilascaríase humana. Sobre três casos encontrados no Distrito Federal, Brasil. Revista do Instituto de Medicina Tropical, v.34, n.16, p. 587-591, 1992. https://doi.org/10.1590/S0036-46651992000600014

VIEIRA, M.A et al. Relato de caso de Lagochilascariose humana procedente do Estado do Pará, Brasil. Revista da Sociedade Brasileira de Medicina Tropical, v.33, n.1, p. 87-90, 2000. https://doi.org/10.1590/S0037-86822000000100014 